\title{
Une bonne mise au point sur un problème majeur
}

\section{Jean Martin}

Dr med., membre de la rédaction

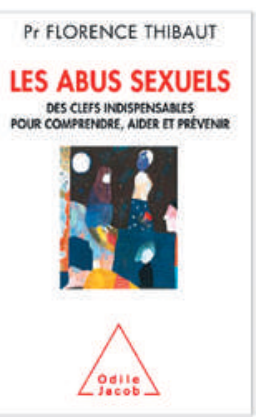

Florence Thibaut Les abus sexuels

Des clefs indispensables pour comprendre, aider et prévenir.

Paris: Editions Odile Jacob; 2015.

240 pages.

40.30 CHF.

ISBN 978-2738131867
«L'histoire de la violence sexuelle remonte aux origines de l'humanité, il a pourtant fallu attendre [jusqu'à récemment] pour que les victimes, en très grande majorité des femmes ou des fillettes, voient leur statut de victimes puis leur souffrance psychologique davantage reconnus.» «On se heurte au mur du silence des victimes; moins de la moitié d'entre elles parlent de leur agression et seulement $10 \%$ osent porter plainte. Elles continuent trop souvent à endosser la culpabilité du viol [...] C'est pourquoi il est très important de laisser une large place à leur parole.» Florence Thibaut, professeur de psychiatrie et d'addictologie à l'Hôpital Cochin, à Paris, s'adresse dans cet ouvrage aux besoins correspondants.

Onze chapitres. Les premiers font un état des lieux, donnant un aperçu historique, des définitions, des indications du registre anthropo-sociologique, puis un panorama de la violence sexuelle en termes chiffrés. Discutant les raisons qui font que les victimes restent si souvent muettes et que les professionnels de santé ou sociaux ne déclarent pas systématiquement les situations. Est présentée une typologie des agresseurs et des diverses paraphilies. A partir du chapitre 4, on entre dans la pratique: quand faut-il penser à une agression, chez un enfant, un adolescent? Le chapitre 6 est consacré au point de vue des victimes et le suivant à leurs réactions psychologiques, immédiates et/ ou à long terme, et à leur prise en charge par diverses méthodes. Viennent ensuite la législation et les aspects judiciaires, avec le renforcement récent des droits de l'enfant et de sa protection, puis l'aide aux victimes. Le dernier chapitre est consacré à la prévention, y compris l'identification précoce des situations à risque et la problématique Internet. Plusieurs annexes pratiques: sur les modalités de signalement, le secret médical auquel il peut être dérogé, l'examen médical et le recueil des preuves. NB: Garder à l'esprit l'importance des certificats médicaux, qui doivent rapporter aussi fidèlement que possible les paroles de la victime (entre guillemets), mais ne doivent faire état par ailleurs que de faits objectivement constatés.

Des points à noter. La survenue d'abus ne dépend pas de manière notable du milieu social. Le viol entre époux est maintenant condamnable dans la plupart des pays.
L'importance aggravante d'un rapport d'autorité ou de dépendance entre abuseur et victime est unanimement reconnue. Le risque est accru de devenir un abuseur si l'on a été soi-même abusé.

S'agissant de pédophilie: «dans la plupart des cas, les signes sont indirects et très peu spécifiques. Ce qui doit alerter, c'est une modification importante du comportement de l'enfant, dans son milieu familial ou scolaire, ne pouvant être expliquée par un évènement identifiable.» Thibaut aborde aussi la question de la crédibilité de l'enfant.

Places respectives du soin et de la justice. Importance de la réparation. "Le thérapeute ne peut pas faire l'impasse sur l'importance, au plan symbolique et dans la réalité, de la réparation judiciaire [...] Si la justice ne s'attache qu'aux éléments factuels, la médecine en revanche se doit de considérer, au-delà des faits, l'immense souffrance de la personne.»

«Difficile pour la victime de faire un choix, sachant que se taire ou parler peut aboutir à la même conséquence: la mort sociale.» Pour l'inceste, des auteurs parlent d'assassinat psychique. On sait la difficulté écrasante, en cas d'inceste, de briser ce "secret entre nous» (abuseur dixit), vu comme une trahison.

Des programmes nécessaires. 1) La mise en place de centres pluridisciplinaires d'accueil et d'examen (pôles de référence); c'est heureusement un domaine où des progrès significatifs sont réalisés, sous l'égide souvent de la médecine légale; 2 ) «les soins apportés aux agresseurs sexuels eux-mêmes sont un élément clef dans la réduction du nombre d'abus.» La formation dans ce but d'équipes compétentes permettra de limiter les récidives (un tel lieu, le Centre prévention de l'Ale, a été ouvert à Lausanne au printemps 2016); 3) un rappel qui devrait aller de soi: «il est très important, pour prévenir, de renforcer les programmes d'éducation sexuelle, et d'insister sur la nécessité d'un consentement entre partenaires lors d'une relation.»

En résumé: sous une forme compréhensible et maniable, un ouvrage présentant l'essentiel des informations utiles sur une problématique majeure et les manières de l'identifier, de la prendre en charge et, dans toute la mesure du possible, de la prévenir. 